\title{
A CONSTRUCTIVE ERGODIC THEOREM
}

\author{
'BY \\ J. A. NUBER( $\left.{ }^{1}\right)$
}

\begin{abstract}
As discussed by Bishop, Birkhoff's Ergodic Theorem is not constructively valid. In this paper we present an hypothesis which is necessary and sufficient for the constructive almost everywhere convergence of the Césaro averages of the translates of an integrable function by a measure preserving transformation. In addition necessary and sufficient conditions are given for the limit function to be constructively integrable. Also we present a necessary and sufficient condition that the averages converge to a constant function and give an equivalent formulation of this condition for finite measure spaces. Several interesting examples are given which satisfy these conditions.
\end{abstract}

Introduction. As shown in [1, pp. 232-233], Birkhoff's Ergodic Theorem is not constructively valid. In the same reference it was shown that the sequence of averages do satisfy, however, certain inequalities (first introduced by Doob in the study of martingales and called upcrossing inequalities). These inequalities (essentially generalizations of Hopf's Maximal Ergodic Inequality) are a good equalhypothesis substitute for the Birkhoff Theorem. That is, under the hypotheses of the Birkhoff Theorem, the upcrossing inequalities are constructively true and these inequalities classically imply with an easy argument the conclusion of the Birkhoff Theorem. Yet, as discussed in [2], there remains the important problem of providing an equal-conclusion substitute for the Birkhoff Theorem. That is, we seek hypotheses such that, in the presence of the original hypotheses of the Birkhoff Theorem, they are satisfied classically in a trivial fashion and they constructively imply the conclusion of the Birkhoff Theorem.

The purpose of this paper is to present a condition on measure preserving transformations, which is a necessary and sufficient condition that the conclusion of Birkhoff's Theorem be constructively true. This condition is shown to be trivially satisfied classically and it is shown to be satisfied constructively in a number of important examples.

In addition a constructively necessary and sufficient condition is given that the

Received by the editors September 18, 1970 and, in revised form, April 19, 1971.

AMS 1969 subject classifications. Primary 2870; Secondary 0223, 6030.

Key words and phrases. Birkhoff's Ergodic Theorem, measure preserving transformation, constructive mathematics, almost everywhere convergence, Césaro averages, integrable function.

(1) This paper represents a portion of the author's doctoral dissertation, directed by Professor Errett Bishop at the University of California at San Diego. The author is indebted to Professor Bishop for his invaluable advice and encouragement.

Copyright (C) 1972, American Mathematical Society 
limit function be in $L_{1}$, this condition being also satisfied classically in a trivial fashion.

All statements of this paper, unless otherwise noted, are to be interpreted in the constructive sense. The necessary background is provided by [1].

1. Principal results.

1. Measure preserving transformations. Throughout $X$ will be a measure space whose measure we denote by $\mu$. An integrable subset $A$ of $X$ will be called null if $\mu(A)=0$ and will be called full if $-A$, the complement of $A$, is null. Two measurable sets $A$ and $B$ are equal, $A=B$, if they differ by a null set.

With these conventions in mind a measure preserving transformation $T$ is a function whose domain $D_{T}$ is a full subset of $X$ and which takes values in $X$ in such a way that

(i) if $A$ is a full subset of $X$ then the set $\left\{x \in D_{T}: T x \in A\right\}$ is a full subset of $X$,

(ii) and if $f$ is any integrable function on $X$, the function $f \circ T$ [which, by (i), is defined almost everywhere according to the rule $f \circ T(x)=f(T x)]$ is also integrable and $\int f \circ T d \mu=\int f d \mu$.

In particular, specializing (ii) to the characteristic function $I_{A}$ of an integrable set $A$, we require that the set $T^{-1} A=\{x: T x \in A\}$ be integrable and

$$
\mu\left(T^{-1} A\right)=\int I_{A} \circ T d \mu=\int I_{A} d \mu=\mu(A) .
$$

In addition we must require

(iii) that for every integrable set $A$ the set $T A=\{T x: x \in A\}$ is integrable.

And (iii) implies, for every integrable $A, \mu(T A)=\mu\left(T^{-1} T A\right) \geqq \mu(A)$ since, up to a null set, $T^{-1} T A \supset A$.

The proof of the next proposition is the main result of this paper.

Proposition. Let $T$ be a measure preserving transformation on the measure space $X$. Then the following two statements are equivalent constructively:

(1.1) For each integrable $f$, the sequences of averages

$$
f^{n}(x)=(n+1)^{-1}\left(f(x)+f(T x)+\cdots+f\left(T^{n} x\right)\right)
$$

converges for almost all $x$ in $X$ as $n \rightarrow \infty$.

(1.2) For each pair $A$ and $B$ of integrable subsets of $X$ and for each $\varepsilon>0$ there are disjoint partitions

$$
A=A_{0} \cup A_{1} \cup \cdots \cup A_{n} \cup A_{*}, \quad-B=B_{0} \cup B_{1} \cup \cdots \cup B_{n} \cup B_{*}
$$

and integers $\{t(1), t(2), \ldots, t(n)\}$ such that $A_{0}, \ldots, A_{n}$ and $B_{0}, \ldots, B_{n}$ are all integrable; such that $T^{t(k)} A_{k}=B_{k}$ for $k=1, \ldots, n$; such that the sets $A_{*} \cap T^{j} B_{*}=\varnothing$ for all integers $j$; and such that $\mu\left(A_{0}\right)+\mu\left(B_{0}\right)<\varepsilon$.

It is perhaps wise, at this point, to prove that condition (1.2) is always satisfied classically in a trivial fashion. 
Suppose $T$ is a measure preserving transformation and $A$ and $B$ are integrable subsets. Define integrable subsets $\left\{A_{k}:-\infty<k<+\infty\right\}$ of $A$ and $\left\{B_{k}:-\infty<k<+\infty\right\}$ of $-B$ inductively in the following fashion. If $n \geqq 0$ is an integer and the sets $\left\{A_{k}:|k|<n\right\}$ and $\left\{B_{k}:|k|<n\right\}$ have been constructed, define the sets $A_{N}^{\prime}=A$ $-\bigcup\left\{A_{k}:|k|<n\right\}$ and $B_{N}^{\prime}=-B-\bigcup\left\{B_{k}^{\prime}:|k|<n\right\}$. Now define

$$
\begin{aligned}
A_{n} & =A_{n}^{\prime} \cap T^{n} B_{n}^{\prime}, & B_{n} & =T^{-n} A_{n}, \\
B_{-n} & =T^{n}\left(A_{n}^{\prime}-A_{n}\right) \cap\left(B_{n}^{\prime}-B_{n}\right), & A_{-n} & =T^{-n} B_{-n} .
\end{aligned}
$$

Since $A$ is integrable, the sets $\left\{A_{k}^{\prime}: k \geqq 0\right\}$ are all integrable, and the sequence $\left\{\mu\left(A_{k}^{\prime}\right): k \geqq 0\right\}$ is a bounded nonincreasing sequence of real numbers which has, classically, a limit. This limit is the measure of the therefore integrable set $\bigcap\left\{A_{k}^{\prime}: k \geqq 0\right\}$. Denote this intersection by $A_{*}$ and denote the intersection $\bigcap\left\{B_{k}^{\prime}: k \geqq 0\right\}$ by $B_{*}$.

Now let $\varepsilon>0$ and choose the integer $N \geqq 0$ so that $\mu\left(A_{N}^{\prime}-A_{*}\right)<\varepsilon / 2$. Since $T$ is measure preserving the sets $A_{k}$ and $B_{k}$ have equal measure, for each integer $k$. Consequently

$$
\mu\left(A_{N}^{\prime}-A_{*}\right)=\sum\left\{\mu\left(A_{k}\right):|k| \geqq N\right\}=\sum\left\{\mu\left(B_{k}\right):|k| \geqq N\right\}=\mu\left(B_{N}^{\prime}-B_{*}\right)
$$

and it follows that the partitions

$$
\begin{aligned}
A & =\left(A_{N}^{\prime}-A_{*}\right) \cup\left\{A_{k}:|k|<N\right\} \cup A_{*}, \\
-B & =\left(B_{N}^{\prime}-B_{*}\right) \cup\left\{B_{k}:|k|<N\right\} \cup B_{*}
\end{aligned}
$$

together with the integers $\{k:-N<k<N\}$ have the properties required by (1.2).

This procedure does not work constructively because the requisite limit may fail to exist constructively. (See for example [1, p. 233].)

As a simple example where (1.2) is satisfied constructively, let $X$ be a locally compact topological group with left invariant Haar measure $\mu$. Let $t$ be an element of $X$ such that for every pair of compact subsets $K$ and $K^{\prime}$ of $X$ there is an integer $k$ for which $K$ and $t^{k} K^{\prime}$ are disjoint. Let $T$ be left translation by $t, T x=t x(x$ in $X)$.

Let $A$ and $B$ be integrable subsets of $X$ and let $\varepsilon>0$. Since $A$ and $B$ are integrable there are compact subsets $K^{\prime} \subset A$ and $K \subset B$ of $X$ such that $\mu\left(A-K^{\prime}\right)+\mu(B-K)<\varepsilon$. Let $k$ be any integer for which $K \cap t^{k} K^{\prime}=\varnothing$. Then define

$$
\begin{array}{ll}
A_{1}=K^{\prime}-T^{-k} B, & B_{1}=T^{k} A_{1}, \\
A_{0}=\left(A-A_{1}\right), & B_{0}=\varnothing, \\
A_{*}=\varnothing, & B_{*}=-B-B_{1} .
\end{array}
$$

Since $t^{k} K^{\prime} \subset-K$ and $K^{\prime}-A_{1}=K^{\prime} \cap T^{-k} B$ we have

$$
\mu\left(K^{\prime}-A_{1}\right)=\mu\left(T^{k}\left(K^{\prime}-A_{1}\right)\right) \leqq \mu\left(t^{k} K^{\prime} \cap B\right) \leqq \mu(B-K)
$$

and so $\mu\left(A_{0}\right)<\varepsilon$. 
Therefore the partitions

$$
A=A_{0} \cup A_{1} \cup A_{*}, \quad B=B_{0} \cup B_{1} \cup B_{*}
$$

together with the integer $\{k\}$ satisfy the requirements of (1.2).

The preceding example actually satisfies a stronger hypothesis than condition (1.2) and the result is summarized in the following corollary.

COROLlaRY 1. The following conditions on a measure preserving transformation $T$, on a set which has a subset of positive measure, are constructively equivalent:

(2.1) for each integrable $f$ the averages $\left\{f^{(n)}(x)\right\}$ converge for almost all $x$ to a constant,

(2.2) for every pair of integrable sets $A$ and $B$ and every $\varepsilon>0$ there are disjoint partitions

$$
A=A_{0} \cup \cdots \cup A_{n}, \quad-B=B_{0} \cup \cdots \cup B_{n}
$$

and integers $t(1), \ldots, t(n)$ such that $A_{1}, \ldots, A_{n}$ and $B_{1}, \ldots, B_{n}$ are all integrable; the sets $T^{t(k)} A_{k}=B_{k}$ for $k=1, \ldots, n$; and at least one of $A_{0}$ and $B_{0}$ is integrable with measure less than $\varepsilon$.

It would be good to conclude at this point that if a measure preserving transformation satisfying (2.1) and (2.2) then the a.e. limit of the averages $\left\{f^{n}\right\}$ of an integrable function $f$ is the space average of that function. Unfortunately the space itself may not be a constructively integrable set, and even if the space is integrable, the limit function may fail to be constructively integrable. The following corollary is directed toward this problem.

COROllaRY 2. Let T be a measure preserving transformation satisfying (1.1)-(1.2). Then equivalent constructively are:

(3.1) For each integrable $f$, the limit $f^{*}$ of the averages $\left\{f^{n}\right\}$ is integrable.

(3.2) For every increasing sequence $\left\{X_{q}: 0 \leqq q<\infty\right\}$ of integrable subsets whose characteristic functions converge almost everywhere to 1 , the following holds.

For every integrable set $A$ and every $\varepsilon>0$ there is a partition $A=A_{1} \cup A_{2}$, into disjoint integrable sets, for which

(i) there is an integer $q \geqq 0$ such that for all integers $k$ we have $\mu\left(T^{k} A_{1}-X_{q}\right)<\varepsilon$ and

(ii) for all integers $p>0$ there is an integer $n>0$ such that $\mu\left(A_{2}-\bigcup_{|k|<n} T^{k}\left(-X_{p}\right)\right)$ $<\varepsilon$.

Classically the condition (3.2) is trivially true since if $\left\{X_{q}: 0 \leqq q<\infty\right\}$ is any increasing sequence of integrable sets whose union is $X$ then the set

$$
A_{1}=A \cap \bigcup_{q=0}^{\infty} \bigcap_{k=-\infty}^{+\infty} T^{k}\left(X_{q}\right)
$$

is integrable and the partition $A=A_{1} \cup\left(A-A_{1}\right)$ has the properties desired in (3.2) for every $\varepsilon>0$. 
In the event $X$ has finite measure (that is, $X$ is integrable) then (3.2) is trivially true for if $A$ is any integrable set we may take $A_{1}=A$ and $A_{2}=\varnothing$ to get an appropriate partition.

The example preceding Corollary 1 satisfies (3.2) since if $A$ is any integrable set the partition $A_{1}=\varnothing$, and $A_{2}=A$ will always satisfy the needs of (3.2).

In order to discuss some further examples, it is convenient to introduce the following finite measure space version of Corollary 1.

COROLlaRY 3. Let $X$ be a finite measure space and let $T$ be a measure preserving transformation on $X$. Then constructively equivalent are

(4.1) For every integrable $f$, the sequence $\left\{f^{n}\right\}$ of averages converges almost everywhere to $\mu(X)^{-1} \int f d \mu$.

(4.2) There is a nonnegative monotone increasing function $r$ defined for nonnegative real numbers such that

(i) for all $\varepsilon>0$ there is $a \delta>0$ so that $x<\varepsilon$ if $r(x)<\delta$; and

(ii) for every pair of integrable sets $A$ and $B$ and every $\varepsilon>0$ there is anteger $k$ for which $\mu\left(A \cap T^{k} B\right)>r(\mu(A) \mu(B))-\varepsilon$.

As an immediate application of this corollary, let $G$ be a compact topological group with left invariant Haar measure $\mu$, normalized so that $\mu(G)=1$. Let $t$ be an element of $G$ such that the powers $\left\{t^{k}: k=0, \pm 1, \ldots\right\}$ are dense in $G$ and let $T$ be the left translation $T x=t x(x$ in $G)$. Let $A$ and $B$ be integrable subsets of $G$ and let $I_{A}$ and $I_{B}$ denote their respective characteristic functions. Let $\varepsilon>0$ be given. The function $I_{B}(x) I_{A}(y x)$, defined for almost all $(x, y)$ in $G \times G$, is integrable, and therefore, by Fubini's theorem and the unimodularity of $G$,

$$
\mu(A) \mu(B)=\int_{G}\left\{\int_{G} I_{B}(x) I_{A}(y x) d \mu_{x}\right\} d \mu_{y} .
$$

Define $f(y)=\int_{G} I_{B}(x) I_{A}(y x) d \mu_{x}$. The function $f$ is continuous in $G$. Therefore, since the set of powers $\left\{t^{k}: k=0, \pm 1, \ldots\right\}$ is dense in $G$, there exists a finite set $J$ of integers such that min $\left[\left|f\left(t^{k}\right)-f(y)\right|: k \in J\right]<\varepsilon / 2$ for all $y$ in $G$. Choose $\varepsilon^{\prime}$ with $\varepsilon>\varepsilon^{\prime}>\varepsilon / 2$ such that the sets $O_{k}=\left\{y:\left|f\left(t^{k}\right)-f(y)\right|<\varepsilon^{\prime}\right\}, k \in J$, are integrable. Then define, for each $k \in J$, the set $U_{k}=O_{k}-\bigcup\left\{O_{j}: j \in J, j<k\right\}$ and denote its characteristic function by $I_{k}$.

It follows from the definitions that $\sum\left\{I_{k}: k \in J\right\}=1$ almost everywhere and that if $I_{k}(y)=1$ then $\left|f\left(t^{k}\right)-f(y)\right|<\varepsilon^{\prime}$.

As a result,

$$
\left|\sum_{k \in J} f\left(t^{k}\right) I_{k}-f\right| \leqq \sum_{k \in J}\left|f\left(t^{k}\right)-f\right| I_{k}<\varepsilon^{\prime}
$$

almost everywhere. Therefore

$$
\left|\mu(A) \cdot \mu(B)-\sum_{k \in J} f\left(t^{k}\right) \mu\left(U_{k}\right)\right|<\varepsilon^{\prime}<\varepsilon .
$$


This implies

$$
\sum_{k \in J}\left[f\left(t^{k}\right)+\varepsilon-\mu(A) \mu(B)\right] \mu\left(U_{k}\right)>0
$$

and thus there is an integer $k$ such that

$$
\left[f\left(t^{k}\right)+\varepsilon-\mu(A) \mu(B)\right] \mu\left(U_{k}\right)>0 .
$$

Since $f\left(t^{k}\right)=\mu\left(A \cap T^{k} B\right)$ it follows that $\mu\left(A \cap T^{k} B\right)>\mu(A) \mu(B)-\varepsilon$.

Consequently left translation on a compact topological group by an element whose powers are dense satisfies (1.3) with $r(x)=x$.

As another application of Corollary 3, let $n$ be a positive integer and let $Z_{n}=\{0,1, \ldots, n-1\}$. Let $X$ be the Cartesian product of countably many copies of $Z_{n}$. Thus the elements of $X$ are the bilaterally infinite sequences of elements of $Z_{n}$, $x=\left\{\ldots, x_{-1}, x_{0}, x_{1}, \ldots\right\}$.

Define a normalized measure in $Z_{n}: \mu(0)=p_{0}, \ldots, \mu(n-1)=p_{n-1}, \sum p_{i}=1$, and let $\mu$ be the induced product measure on $X$. Let $T$ be translation to the right by 1 :

$$
T x=x^{\prime} \quad \text { where } x_{i}^{\prime}=x_{i-1} .
$$

It is an easy result that $T$ is measure preserving.

For each subset $J$ of integers, let $\mathscr{R}_{J}$ be the algebra of integrable subsets, generated by sets of the form $\left\{x: x_{i}=j\right\}$ for $i$ in $J$ and $j$ in $Z_{n}$. If $J$ and $I$ are disjoint subsets of integers and $A \in \mathscr{R}_{J}$ and $B \in \mathscr{R}_{I}$, then $\mu(A \cap B)=\mu(A) \mu(B)$.

Therefore if $I$ and $J$ are finite subsets of integers and $k$ is so large that $I$ and $\{j: j+k \in J\}$ are disjoint, then

$$
\mu\left(A \cap T^{-k} B\right)=\mu(A) \mu\left(T^{-k} B\right)=\mu(A) \mu(B)
$$

whenever $A \in \mathscr{R}_{I}$ and $B \in \mathscr{R}_{\mathrm{J}}$.

Let $\varepsilon>0$. Let $A$ and $B$ be integrable subsets of $X$. Then there are finite subsets $I$ and $J$ and sets $A^{\prime}$ in $\mathscr{R}_{I}$ and $B^{\prime}$ in $\mathscr{R}_{J}$ such that $\mu\left(A^{\prime} \Delta A\right)+\mu\left(B^{\prime} \Delta B\right)<\varepsilon$. If $k$ is any integer such that $\mu\left(A^{\prime} \cap T^{k} B^{\prime}\right)=\mu\left(A^{\prime}\right) \mu\left(B^{\prime}\right)$, then

$$
\mu\left(A \cap T^{k} B\right)>\mu\left(A^{\prime} \cap T^{k} B^{\prime}\right)-\varepsilon=\mu\left(A^{\prime}\right) \mu\left(B^{\prime}\right)-\varepsilon>\mu(A) \mu(B)-2 \varepsilon .
$$

Therefore the shift transformation on this measure space satisfies (3.2) with $r(x)=x$.

The examples so far described have all satisfied the constructive ergodic condition (2.2). The next corollary will yield examples satisfying the more general (1.2) and not (2.2).

COROLlARY 4. Let $T$ be a measure preserving transformation on a measure space $X$ such that there is a disjoint partition $X=X^{1} \cup X^{2} \cup \cdots \cup X^{n}$ by measurable subsets of $X$ for which

(i) $T^{j} X^{p} \cap X^{q}=\varnothing$ for all integers $j, p$, and $q$ with $p \neq q$, and

(ii) the restriction of $T$ to $X^{p}$ satisfies (1.2) for $p=1, \ldots, n$. 
Then $T$ satisfies (1.2) on $X$, so that for every integrable function $f$ on $X$ the sequence of averages $\left\{f^{n}\right\}$ converges almost everywhere.

As an example of the application of Corollary 2, let $X$ be the disjoint union of two copies of the unit circle. Let $T$ be the measure preserving transformation on $X$ which on one copy of the unit circle is the rotation by $2 \pi \alpha_{1}$ and on the other is rotation by $2 \pi \alpha_{2}$ where both $\alpha_{1}$ and $\alpha_{2}$ are irrational. Then $T$, satisfying (1.3) on each copy of the unit circle, satisfies (1.1) on their union, $X$. More interesting examples could presumably be obtained by replacing the finite decomposition of $X$ in Corollary 4 by a countable, or even continuous, decomposition.

2. Upcrossings. Before proceeding to the proof of the proposition, it is necessary to introduce some conventions, some notation, and some results concerning upcrossings.

It is proven in [1] that if $g$ is a measurable function, then for all except countably many real numbers $\alpha$ (the exceptional values) there is a measurable set $A$ such that $g<\alpha$ (a.e.) on $A$ and $g \geqq \alpha$ (a.e.) on $-A$. In the interests of simplicity, the following conventions treating this situation are adopted. If $g$ is a measurable function, the notation $\{x: g(x)<\alpha\}$ will mean that $A \equiv\{x: g(x)<\alpha\}$ is a measurable set such that $g<\alpha$ (a.e.) on $A$ and $g \geqq \alpha$ (a.e.) on $-A$. Statements containing phrases of the form

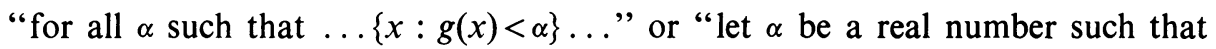
$\ldots\{x: g(x)<\alpha\} \ldots$..." will implicitly assume all exceptional values of $\alpha$ are omitted.

Let $n$ be a positive integer and let $\left\{a_{1}, \ldots, a_{n}\right\}$ be a sequence of real numbers. For every pair of real numbers $\alpha<\beta$, the number of upcrossings of the sequence $\left\{a_{1}, \ldots, a_{n}\right\}$ over the interval $(\alpha, \beta)$ is defined to be the maximum integer $h$ (if it exists) such that there are integers $1 \leqq u(1)<v(1)<\cdots<u(h)<v(h) \leqq n$ for which $a_{u(k)} \leqq \alpha$ and $a_{v(k)} \geqq \beta$ for $k=1, \ldots, h$.

Let $\left\{f_{k}: 0 \leqq k<\infty\right\}$ be a sequence of measurable functions and $\alpha<\beta$ be real numbers. Let $f_{-1} \equiv \alpha$. Let $n \geqq-1$ and $m \geqq 0$ be integers. Then for almost all $x$ the maximum integer $h$ such that there are integers $n \leqq u(1)<v(1)<\cdots<u(h)<v(h)$ $\leqq m+n$ with

$$
x \in \bigcap_{j=1}^{h}\left\{x: f_{u(j)}(x) \leqq \alpha\right\} \cap\left\{x: f_{v(j)}(x) \geqq \beta\right\}
$$

is well defined. Denote this maximum integer $h$ by $U_{n, m}\left(x \mid\left\{f_{k}\right\}, \alpha, \beta\right)$.

Let $T$ be a positive operator from $L_{1}$ to $L_{1}$ which is bounded by 1 . Let

$$
\left\{f_{j}: j=0,1, \ldots\right\}
$$

be a sequence of measurable functions such that $f_{j}^{+}$is integrable for each $j$ and

$$
T\left(\sum_{j \in \Omega} f_{j}\right)^{+} \geqq \sum_{j \in \Omega} f_{j+1} \quad \text { (a.e.) }
$$

for each finite subset $\Omega$ of nonnegative integers. For each nonnegative integer $n$ write $f^{n} \equiv(n+1)^{-1}\left(f_{0}+f_{1}+\cdots+f_{n}\right)$. 
In [1], a general result is proven which specializes in the present case to

TheOREM (UPCROSSING INEQUALITY). With the above definitions, $U_{n, m}\left(x \mid\left\{f^{k}\right\}, \alpha, \beta\right)$ is integrable and

$$
U_{n, m}\left(x \mid\left\{f^{k}\right\}, \alpha, \beta\right) d \mu \leqq \frac{1}{\beta-\alpha} \int\left(f_{0}-\alpha\right)^{+} d \mu .
$$

In particular, the linear operator from $L_{1}$ to $L_{1}$ induced by a measure preserving transformation $T$, together with the sequence $\left\{T^{j} f: j \geqq 0\right\}$ of iterates of a measurable function $f$ with $f^{+}$in $L_{1}$, satisfies the hypothesis of the theorem.

For the purpose here the sequence $\left\{f_{j}\right\}$ will always be a sequence of iterates $\left\{T^{j} f\right\}$ and $U_{n, m}(x \mid f, \alpha, \beta)$ is written in place of $U_{n, m}\left(x \mid\left\{f^{k}\right\}, \alpha, \beta\right)$.

Also, in the sequel, reference to $U_{n, m}(x \mid f, \alpha, \beta)$ will imply $\alpha$ and $\beta$ are real numbers which are not exceptional values for any of the functions $f^{k}$ for $n \leqq k \leqq n+m$.

3. Proof of the proposition and corollaries. The proof proceeds through a series of lemmas, the first of which is a reformulation of a result in [3].

LEMMA 1. Let $T$ be a positive linear operator from $L_{1}$ to $L_{1}$ that is bounded by 1 . Let $\left\{f_{n}\right\}_{n=1}^{\infty}$ be a sequence of uniformly integrable functions which converges to zero in measure. Then $\left\{\max _{0 \leqq \nu \leqq N}\left|f_{n}^{\nu}\right|\right\}_{n=1}^{\infty}$ converges to zero in measure uniformly for nonnegative integers $N$.

Proof. Let $\varepsilon>0$. Let $\delta>0$ be such that if $A$ is integrable and $\mu(A)<\delta$ then $\int_{A}\left|f_{n}\right| d \mu<\varepsilon^{2} / 2$ for each positive integer $n$.

Let $M$ be a positive integer for which $n \geqq M$ implies $\mu\left(\left\{x:\left|f_{n}(x)\right|>\varepsilon / 2\right\}\right)<\delta$. Then if $N$ is any nonnegative integer and $n \geqq M$

$$
\mu\left(\left\{x: \max \left[f_{n}^{\nu}(x): 0 \leqq \nu \leqq N\right] \geqq \varepsilon\right\}\right) \leqq \int U_{-1, N+1}\left(x \mid f_{n}, \varepsilon / 2, \varepsilon\right) d \mu .
$$

Thus, by the Upcrossing Inequality,

$$
\mu\left(\left\{x: \max \left[f_{n}^{\nu}(x): 0 \leqq \nu \leqq N\right]>\varepsilon\right\}\right) \leqq \frac{2}{\varepsilon} \int\left(f_{n}-\frac{\varepsilon}{2}\right)^{+} d \mu \leqq \frac{2}{\varepsilon} \int_{A} f_{n} d \mu
$$

where $A=\left\{x: f_{n}(x)>\varepsilon / 2\right\}$. Similarly, for each nonnegative integer $N$ and integer $n \geqq M$,

$$
\begin{aligned}
\mu\left(\left\{x: \min \left[f_{n}^{\nu}(x): 0\right.\right.\right. & \leqq \nu \leqq N] \leqq-\varepsilon\}) \\
& =\mu\left(\left\{x: \max \left[\left(-f_{n}\right)^{\nu}(x): 0 \leqq \nu \leqq N\right] \geqq \varepsilon\right\}\right) \leqq \frac{2}{\varepsilon} \int_{A^{\prime}}\left(-f_{n}\right) d \mu
\end{aligned}
$$

where $A^{\prime}=\left\{x:-f_{n}(x)>\varepsilon / 2\right\}$. Putting these two together yields

$$
\mu\left(\left\{x: \max \left[\left|f_{n}^{\nu}(x)\right|: 0 \leqq \nu \leqq N\right] \geqq \varepsilon\right\}\right) \leqq \frac{2}{\varepsilon} \int_{A \cup A^{\prime}}\left|f_{n}\right| d \mu<\frac{2}{\varepsilon} \frac{\varepsilon^{2}}{2}=\varepsilon
$$

for each nonnegative integer $N$ and integer $n \geqq M$. 
Lemma 2. Let $f$ be a bounded nonnegative integrable function. Then, under the hypothesis (1.2), for each pair of real numbers $\alpha$ and $\beta$, with $0<\alpha<\beta$, and for each $\varepsilon>0$ there is a nonnegative integer $N$ such that there are disjoint partitions

$$
\left\{x: f^{N}(x) \geqq \beta\right\}=A_{c} \cup A_{*}, \quad\left\{x: f^{N}(x) \leqq \alpha\right\}=B_{c} \cup B_{*}
$$

for which $A_{c}$ and $B_{c}$ are integrable and $\mu\left(A_{c}\right)+\mu\left(B_{c}\right)<\varepsilon$, and $A_{*} \cap T^{j} B_{*}=\varnothing$ for every integer $j$.

Proof. Let $\alpha, \beta$, and $\lambda$ be real numbers such that $0<\alpha<\beta$ and $0<\lambda<\frac{1}{2}(\beta-\alpha)$. Let $m$ be a nonnegative integer and let $\varepsilon>0$. Define the sets

$$
\bar{A}=\left\{x: f^{m}(x) \geqq \beta-\lambda\right\}, \quad \bar{B}=\left\{x: f^{m}(x) \leqq \alpha+\lambda\right\} .
$$

By hypothesis (1.2) on $T$, there are disjoint partitions $\bar{A}=\bar{A}_{0} \cup \cdots \cup \bar{A}_{v} \cup \bar{A}_{*}$ and $\bar{B}=\bar{B}_{0} \cup \ldots \cup \bar{B}_{v} \cup \bar{B}_{*}$ and integers $v(1), \ldots, v(r)$ such that the $\left\{\bar{A}_{k}\right\}$ and $\left\{\bar{B}_{k}\right\}$ are integrable, $\mu\left(\bar{A}_{0}\right)+\mu\left(\bar{B}_{0}\right)<\varepsilon$, for every integer $j$ the set $\bar{A}_{*} \cap T^{j} \bar{B}_{*}=\varnothing$, and $T^{v(k)} A_{k}=B_{k}$ for $k=1, \ldots, r$.

Set $t_{0}=\max [|v(k)|: k=1, \ldots, r]$ and let $M$ be a bound for $f$. Choose $n>m$ so large that $2 t_{0} M /(n+1) \leqq \lambda$. Then for each integer $t$ with $1 \leqq t \leqq t_{0}$,

$$
\left|T^{t} f^{n}(x)-f^{n}(x)\right| \leqq \frac{1}{n+1}\left|\sum_{k=n+1}^{n+t} f\left(T^{k} x\right)-\sum_{k=0}^{t-1} f\left(T^{k}(x)\right)\right| \leqq \frac{2 t_{0} M}{n+1} \leqq \lambda .
$$

Thus,

$$
\mu\left(T^{v(k)}\left\{x: f^{n}(x) \geqq \beta\right\}-\left\{x: f^{n}(x) \geqq \beta-\lambda\right\}\right)=0
$$

and

$$
\mu\left(T^{-v(k)}\left\{x: f^{n}(x) \leqq \alpha\right\}-\left\{x: f^{n}(x) \leqq \alpha+\lambda\right\}\right)=0
$$

for $k=1, \ldots, r$.

Define the sets

$$
A=\left\{x: f^{n}(x) \geqq \beta\right\}, \quad B=\left\{x: f^{n}(x) \leqq \alpha\right\} .
$$

Again by the hypothesis (1.1) on $T$, there are disjoint partitions, $A=A_{0} \cup \cdots \cup A_{s}$ $\cup A_{*}, B=B_{0} \cup \ldots \cup B_{s} \cup B_{*}$ and integers $u(1), \ldots, u(s)$ such that the $\left\{A_{k}\right\}$ and $\left\{B_{k}\right\}$ are integrable, $\mu\left(A_{0}\right)+\mu\left(B_{0}\right)<\varepsilon / 3$, for every integer $j$ the set $A_{*} \cap T^{j} B_{*}=\varnothing$, and $T^{u(k)} A_{k}=B_{k}$ for $k=1, \ldots, s$. Define the sets

$$
\begin{array}{ll}
A_{c} \equiv A-A_{*}=\bigcup_{k=0}^{s} A_{k}, & B_{c} \equiv B-B_{*}=\bigcup_{k=0}^{s} B_{k}, \\
\bar{A}_{c} \equiv \bar{A}-\bar{A}_{*}=\bigcup_{k=0}^{r} \bar{A}_{k}, & \bar{B}_{c} \equiv \bar{B}-\bar{B}_{*}=\bigcup_{k=0}^{r} \bar{B}_{k} .
\end{array}
$$

Estimating $\mu\left(A_{c}\right)+\mu\left(B_{c}\right)$ in terms of the sets $\bar{A}$ and $\bar{B}$ gives

$$
\mu\left(A_{c}\right)+\mu\left(B_{c}\right)=\mu\left(A_{c} \cap \bar{A}\right)+\mu\left(A_{c}-\bar{A}\right)+\mu\left(B_{c} \cap \bar{B}\right)+\mu\left(B_{c}-\bar{B}\right) .
$$


Now

$$
\mu\left(A_{c} \cap \bar{A}\right)+\mu\left(B_{c} \cap \bar{B}\right)=\mu\left(A_{c} \cap \bar{A}_{c}\right)+\mu\left(A_{c} \cap \bar{A}_{*}\right)+\mu\left(B_{c} \cap \bar{B}_{c}\right)+\mu\left(B_{c} \cap \bar{B}_{*}\right) .
$$

Since

$$
\begin{aligned}
\mu\left(A_{c} \cap \bar{A}_{*}\right)+\mu\left(B_{c} \cap \bar{B}_{*}\right) & =\sum_{k=0}^{s}\left[\mu\left(A_{k} \cap \bar{A}_{*}\right)+\mu\left(B_{k} \cap \bar{B}_{*}\right)\right] \\
& \leqq \sum_{k=1}^{s}\left[\mu\left(T^{u(k)}\left(A_{k} \cap \bar{A}_{*}\right)\right)+\mu\left(T^{-u(k)}\left(B_{k} \cap \bar{B}_{*}\right)\right)\right]+\frac{\varepsilon}{3} \\
& \leqq \sum_{k=1}^{s}\left[\mu\left(B_{k} \cap T^{u(k)} \bar{A}_{*}\right)+\mu\left(A_{k} \cap T^{-u(k)} \bar{B}_{*}\right)\right]+\frac{\varepsilon}{3} \\
& \leqq \sum_{k=1}^{s}\left[\mu\left(B_{k}-\bar{B}_{*}\right)+\mu\left(A_{k}-\bar{A}_{*}\right)\right]+\frac{\varepsilon}{3} \\
& \leqq \mu\left(B_{c}-\bar{B}_{*}\right)+\mu\left(A_{c}-\bar{A}_{*}\right)+\frac{\varepsilon}{3}
\end{aligned}
$$

it follows that

$$
\begin{aligned}
\mu\left(A_{c} \cap \bar{A}\right)+\mu\left(B_{c} \cap \bar{B}\right) & \leqq \mu\left(B_{c} \cap \bar{B}_{c}\right)+\mu\left(A_{c} \cap \bar{A}_{c}\right)+\mu\left(B_{c}-\bar{B}_{*}\right)+\mu\left(A_{c}-\bar{A}_{*}\right)+\varepsilon / 3 \\
& \leqq 2\left\{\mu\left(B_{c} \cap \bar{B}_{c}\right)+\mu\left(A_{c} \cap \bar{A}_{c}\right)\right\}+\mu\left(B_{c}-\bar{B}\right)+\mu\left(A_{c}-\bar{A}\right)+\varepsilon / 3 .
\end{aligned}
$$

Therefore

(1) $\mu\left(A_{c}\right)+\mu\left(B_{c}\right) \leqq 2\left\{\mu\left(B_{c}-\bar{B}\right)+\mu\left(A_{c}-\bar{A}\right)+\mu\left(A_{c} \cap \bar{A}_{c}\right)+\mu\left(B_{c} \cap \bar{B}_{c}\right)\right\}+\varepsilon / 3$.

Now

$$
\mu\left(B_{c}-\bar{B}\right)+\mu\left(A_{c}-\bar{A}\right) \leqq \mu(B-\bar{B})+\mu(A-\bar{A})
$$

and

$$
\begin{aligned}
\mu\left(A_{c} \cap \bar{A}_{c}\right) & +\mu\left(B_{c} \cap \bar{B}_{c}\right)=\sum_{k=0}^{r}\left[\mu\left(A_{c} \cap \bar{A}_{k}\right)+\mu\left(B_{c} \cap \bar{B}_{k}\right)\right] \\
& \leqq \sum_{k=1}^{r}\left[\mu\left(T^{v(k)}\left(A_{c} \cap \bar{A}_{k}\right)\right)+\mu\left(T^{-v(k)}\left(B_{c} \cap \bar{B}_{k}\right)\right)\right]+\frac{\varepsilon}{3} \\
& \leqq \sum_{k=1}^{r}\left[\mu\left(T^{v(k)} A_{c} \cap \bar{B}_{k}\right)+\mu\left(T^{-v(k)} B_{c} \cap \bar{A}_{k}\right)\right]+\frac{\varepsilon}{3} \\
& \leqq \sum_{k=1}^{r}\left[\mu\left(T^{v(k)}\left\{x: f^{n}(x) \geqq \beta\right\} \cap \bar{B}_{k}\right)+\mu\left(T^{-v(k)}\left\{x: f^{n}(x) \leqq \alpha\right\} \cap \bar{A}_{k}\right)\right]+\frac{\varepsilon}{3} \\
& \leqq \sum_{k=1}^{r}\left[\mu\left(\left\{x: f^{n}(x) \geqq \beta-\lambda\right\} \cap \bar{B}_{k}\right)+\mu\left(\left\{x: f^{n}(x) \leqq \alpha+\lambda\right\} \cap \bar{A}_{k}\right)\right]+\frac{\varepsilon}{3} \\
& \leqq \mu\left(\left\{x: f^{n}(x) \geqq \beta-\lambda\right\} \cap \bar{B}\right)+\mu\left(\left\{x: f^{n}(x) \leqq \alpha+\lambda\right\} \cap \bar{A}\right)+\varepsilon / 3 .
\end{aligned}
$$

Define

$$
\begin{aligned}
S(m, n)= & \mu(A-\bar{A})+\mu(B-\bar{B})+\mu\left(\left\{x: f^{n}(x) \geqq \beta-\lambda\right\} \cap \bar{B}\right) \\
& +\mu\left(\left\{x: f^{n}(x) \leqq \alpha+\lambda\right\} \cap \bar{A}\right) .
\end{aligned}
$$

It follows from (1), (2) and (3) that $\mu\left(A_{c}\right)+\mu\left(B_{c}\right) \leqq 2 S(m, n)+\varepsilon$. 
These estimations have given us the following result:

(4) for each nonnegative integer $m$ and for each $\varepsilon>0$ there is an integer $n>m$ such that there are disjoint partitions

$$
\left\{x: f^{n}(x) \geqq \beta\right\}=A_{c} \cup A_{*}, \quad\left\{x: f^{n}(x) \leqq \alpha\right\}=B_{c} \cup B_{*}
$$

where $A_{c}$ and $B_{c}$ are integrable, $\mu\left(A_{c}\right)+\mu\left(B_{c}\right)<2 S(m, n)+\varepsilon$, and for each integer $j$ we have $A_{*} \cap T^{j} B_{*}=\varnothing$.

To complete the proof of this lemma let $\varepsilon>0$ and use the result (4) inductively to obtain a sequence

$$
0=n(0)<n(1)<\cdots<n(k)<\cdots
$$

of integers such that for $k=1,2, \ldots$ there are disjoint partitions

$$
\left\{x: f^{n(k)}(x) \geqq \beta\right\}=A_{c}^{k} \cup A_{*}^{k}, \quad\left\{x: f^{n(k)}(x) \leqq \alpha\right\}=B_{c}^{k} \cup B_{*}^{k}
$$

for which $A_{c}^{k}$ and $B_{c}^{k}$ are integrable and $\mu\left(A_{c}^{k}\right)+\mu\left(B_{c}^{k}\right)<2 S(n(k-1)+1, n(k))+\varepsilon / 2$ and $A_{*}^{k} \cap T^{j} B_{*}^{k}=\varnothing$ for each integer $j$.

Let $K$ be an integer so large that

$$
4\left[\frac{1}{\lambda}+\frac{1}{(\beta-\alpha-2 \lambda)}\right] \int f d \mu<K \cdot \varepsilon / 2 .
$$

Now

Since

$$
\sum_{k=1}^{K}\left[\mu\left(A_{c}^{k}\right)+\mu\left(B_{c}^{k}\right)-\varepsilon\right] \leqq \sum_{k=1}^{K}[2 S(n(k-1)+1, n(k))-\varepsilon / 2]
$$

$$
\begin{aligned}
\sum_{k=1}^{K} S(n(k-1)+1, & n(k)) \\
\leqq & \int U_{0, n(K)}(x \mid f, \beta-\lambda, \beta) d \mu+\int U_{-1, n(K)+1}(x \mid f, \alpha, \alpha+\lambda) d \mu \\
& +\int U_{0, n(K)}(x \mid f, \alpha+\lambda, \beta-\lambda) d \mu+\int U_{-1, n(K)+1}(x \mid f, \alpha+\lambda, \beta-\lambda) d \mu
\end{aligned}
$$

and since $(f-\beta+\lambda)^{+},(f-\alpha-\lambda)^{+}$, and $(f-\alpha)^{+}$are all integrable, by Bishop's Upcrossing Inequality

$$
\begin{aligned}
\sum_{k=1}^{K} S(n(k-1)+1, n(k)) \leqq & \frac{1}{\lambda} \int(f-\beta+\lambda)^{+} d \mu+\frac{1}{\lambda} \int(f-\alpha)^{+} d \mu \\
& +\frac{2}{\beta-\alpha-2 \lambda} \int(f-\alpha-\lambda)^{+} d \mu \\
\leqq & 2\left[\frac{1}{\lambda}+\frac{1}{\beta-\alpha-2 \lambda}\right] \int f d \mu .
\end{aligned}
$$

Therefore

$$
\sum_{k=1}^{K}\left(\mu\left(A_{c}^{k}\right)+\mu\left(B_{c}^{k}\right)-\varepsilon\right) \leqq 4\left[\frac{1}{\lambda}+\frac{1}{\beta-\alpha-2 \lambda}\right] f d \mu-K \frac{\varepsilon}{2}<0 .
$$


This implies there is an integer $k$ with $1 \leqq k \leqq K$ such that $\mu\left(A_{c}^{k}\right)+\mu\left(B_{c}^{k}\right)<\varepsilon$. Setting $N=n(k)$, the proof of the lemma is complete.

For each pair of nonnegative integers $n$ and $m$, for each pair of real numbers $\alpha$ and $\beta$ with $\alpha<\beta$ and for each measurable function $f$ define

$$
U_{n, m}^{*}(x \mid f, \alpha, \beta)=\max \left[U_{n, m}(x \mid f, \alpha, \beta), U_{n, m}(x \mid-f,-\beta,-\alpha)\right] .
$$

In the lemmas that follow, a certain property of $U^{*}$ will be important. Let $f$ be a measurable function and let $\alpha, \beta$, and $\lambda$ be real numbers with $\alpha<\beta$ and $0<\lambda$ $<\frac{1}{2}(\beta-\alpha)$. Let $n$ and $m$ be nonnegative integers. Let $g$ be a measurable function and let $x$ in $X$ be such that $\max \left[\left|g^{v}(x)\right|: n \leqq \nu \leqq n+m\right]<\lambda$. Then

$$
U_{n, m}^{*}(x \mid f, \alpha, \beta) \leqq U_{n, m}^{*}(x \mid f-g, \alpha+\lambda, \beta-\lambda) .
$$

Also note that if $U_{n, m}^{*}(x \mid f, \alpha, \beta)$ is measurable then 0 is not an exceptional value since $U_{n, m}^{*}(x \mid f, \alpha, \beta)$ is integer valued.

LEMMA 3. Let $f$ be a bounded nonnegative integrable function. Then, under the hypothesis (1.2), for each pair of real numbers $\alpha$ and $\beta$ with $0 \leqq \alpha<\beta$ there is a subsequence of the sequence $\left\{U_{0, n}^{*}\left(x \mid f^{N}, \alpha, \beta\right)\right\}_{N=1}^{\infty}$ which converges to zero in measure uniformly for nonnegative integer $n$.

Proof. Let $\alpha, \beta$, and $\lambda$ be real numbers such that $0<\lambda<\frac{1}{2}(\beta-\alpha)$. Let $\varepsilon>0$. By Lemma 2, for each positive integer $n$ there is an integer $N(n)$ such that there are disjoint partitions

$$
\left\{x: f^{N(n)}(x) \geqq \beta-\lambda\right\}=A_{c}^{n} \cup A_{*}^{n}
$$

and

$$
\left\{x: f^{N(n)}(x) \leqq \alpha+\lambda\right\}=B_{c}^{n} \cup B_{*}^{n}
$$

for which $A_{c}^{n}$ and $B_{c}^{n}$ are integrable and $\mu\left(A_{c}^{n}\right)+\mu\left(B_{c}^{n}\right)<1 / n$ and $A_{*}^{n} \cap T^{j} B_{*}^{n}=\varnothing$ for each integer $j$.

Define

$$
\begin{aligned}
g_{n}(x) & =f^{N(n)}(x)-\frac{1}{2}(\beta-\alpha) & & \text { if } x \in A_{c}^{n} \cup B_{c}^{n}, \\
& =0 & & \text { if } x \in-\left(A_{c}^{n} \cup B_{c}^{n}\right)
\end{aligned}
$$

for each positive integer $n$.

The sequence $\left\{g_{n}\right\}$ is uniformly integrable (being bounded) and converges to zero in measure.

Let $\lambda^{\prime}$ be a real number with $0<\lambda^{\prime}<\lambda$. Then by Lemma 1 there is an integer $N$ such that for integers $n \geqq N$

$$
\mu\left(\left\{x: \max \left[\left|g_{n}^{v}(x)\right|: 0 \leqq v \leqq m\right] \geqq \lambda^{\prime}\right\}\right)<\varepsilon
$$

for each nonnegative integer $m$. On the set where $\max \left[\left|g_{n}^{v}(x)\right|: 0 \leqq \nu \leqq m\right]<\lambda^{\prime}$, we have

$$
U_{0, m}^{*}\left(x \mid f^{N(n)}, \alpha, \beta\right) \leqq U_{0, m}^{*}\left(x \mid f^{N(n)}-g_{n}, \alpha+\lambda^{\prime}, \beta+\lambda^{\prime}\right) .
$$


Define

$$
\begin{aligned}
h(x)=f^{N(n)}(x)-g_{n}(x) & =(\beta+\alpha) / 2 \quad \text { if } x \in A_{c}^{n} \cup B_{c}^{n}, \\
& =f^{N(n)}(x) \quad \text { if } x \in-\left(A_{c}^{n} \cup B_{c}^{n}\right) .
\end{aligned}
$$

If there are integers $u$ and $v$ with $h^{u}(x) \leqq \alpha+\lambda^{\prime}$ and $h^{v}(x) \geqq \beta-\lambda^{\prime}$, there are integers $r$ and $s$ with $h\left(T^{r} x\right)>\beta-\lambda$ and $h\left(T^{s} x\right)<\alpha+\lambda$. With $h(x)$ defined as it is, this would imply $T^{r} x \in A_{*}^{n}$ and $T^{s} x \in B_{*}^{n}$. Since $A_{*}^{n} \cap T^{j} B_{*}^{n}=\varnothing$ for all integers $j$, it follows that $U_{0, m}\left(x \mid h, \alpha+\lambda^{\prime}, \beta-\lambda^{\prime}\right)=0$ (a.e.) for each nonnegative integer $m$. Therefore,

$$
\begin{aligned}
\mu\left(\left\{x: U_{0, m}^{*}\left(x \mid f^{N(n)}, \alpha, \beta\right)>0\right\}\right) \leqq & \mu\left(\left\{x: \max \left[\left|g_{n}^{v}(x)\right|: 0 \leqq v \leqq m\right] \geqq \lambda^{\prime}\right\}\right) \\
& +\mu\left(\left\{x: U_{0, m}^{*}\left(x \mid h, \alpha+\lambda^{\prime}, \beta-\lambda^{\prime}\right)>0\right\}\right)<\varepsilon
\end{aligned}
$$

for each nonnegative integer $m$.

LEMMA 4. Let $f$ be a bounded nonnegative integrable function. Let $\alpha$ and $\beta$ be real numbers with $0 \leqq \alpha<\beta$. Then, under the hypothesis (1.2),

$$
\lim _{n \rightarrow \infty} \mu\left(\left\{x: U_{n, m}^{*}(x \mid f, \alpha, \beta)>0\right\}\right)=0
$$

uniformly for nonnegative integers $m$.

Proof. For each positive integer $n, f$ can be written $f=f^{n}+g_{n}-g_{n} \circ T$ where $g_{n}$ is defined for almost all $x$ in $X$ by

$$
g_{n}(x)=\sum_{k=0}^{n-1} \frac{n-k}{n+1} f\left(T^{k} x\right)
$$

Let $\lambda$ be a real number with $0<\lambda<\frac{1}{2}(\beta-\alpha)$. Let $\varepsilon>0$. By Lemma 3, there is an integer $N$ such that

$$
\mu\left(\left\{x: U_{0, n}^{*}\left(x \mid f^{N}, \alpha+\lambda, \beta-\lambda\right)>0\right\}\right)<\varepsilon
$$

for each nonnegative integer $n$.

Since $g_{N}$ is bounded, there is a nonnegative integer $n_{0}$ such that $n \geqq n_{0}$ implies

$$
\left|\left(g_{N}-g_{N} \circ T\right)^{n}(x)\right|=(n+1)^{-1}\left|g_{N}(x)-g_{N}\left(T^{n+1} x\right)\right| \leqq \lambda
$$

for almost all $x$ in $X$.

Then, for each nonnegative integer $m$ and each integer $n \geqq n_{0}$,

$$
U_{n, m}^{*}(x \mid f, \alpha, \beta) \leqq U_{n, m}^{*}\left(x \mid f^{N}, \alpha+\lambda, \beta-\lambda\right) \quad \text { (a.e.). }
$$

Therefore $\mu\left(\left\{x: U_{n, m}^{*}(x \mid f, \alpha, \beta)>0\right\}\right)<\varepsilon$ for each pair of nonnegative integers $n$ and $m$ with $n \geqq n_{0}$. This implies the claim of the lemma.

Proposition. Let $T$ be a measure preserving transformation. Then constructively equivalent are

(1.1) for each integrable function $f$ the averages $\left\{f^{n}\right\}$ converge almost everywhere, and

(1.2) for every pair of integrable sets $A$ and $B$ and every $\varepsilon>0$ there are disjoint measurable partitions $A=A_{0} \cup A_{1} \cup \cdots \cup A_{n} \cup A_{*},-B=B_{0} \cup B_{1} \cup \cdots \cup B_{n} \cup B_{*}$ 
and integers $t(1), \ldots, t(n)$ such that $T^{t(k)} A_{k}=B_{k}(1 \leqq k \leqq n)$ such that $T^{k} A_{*} \cap B_{*}=\varnothing$ for all integers $k$, and such that $\mu\left(A_{0}\right)+\mu\left(B_{0}\right)<\varepsilon$.

Proof. Assume (1.2). Let $f$ be an integrable function. Since $\left\{f^{n}\right\}$ converges almost everywhere if $\left\{\left(f^{+}\right)^{n}\right\}$ and $\left\{\left(f^{-}\right)^{n}\right\}$ converge almost everywhere, it is sufficient to assume $f$ is nonnegative.

Let $\varepsilon>0$ and let $0<\lambda<\frac{1}{2} \varepsilon$.

For each positive integer $n$ the functions $f_{n}(x)=\min [f(x), n], g_{n}(x)=f(x)-f_{n}(x)$ are defined for almost all $x$ in $X$. The sequence $\left\{g_{n}\right\}$ uniformly integrable (being dominated by the integrable function $f$ ) and converges to zero in measure.

By Lemma 1 there is therefore an integer $N_{0}$ such that for integers $N \geqq N_{0}$

$$
\mu\left(\left\{x: \max \left[\left|g_{N}^{\nu}(x)\right|: 0 \leqq \nu \leqq n\right] \geqq \lambda\right\}\right)<\varepsilon / 2
$$

for each nonnegative integer $n$.

Let $\varepsilon^{\prime}=\varepsilon-2 \lambda$. Suppose $f^{n+m}(x)-f^{n}(x) \geqq \varepsilon$ for some nonnegative integers $n$ and $m$ and for some $x$ in $X$ such that $\max \left[\left|g_{N}^{v}(x)\right|: 0 \leqq \nu \leqq n+m\right]<\lambda$. Then $f_{N}^{n+m}(x)$ $-f_{N}^{n}(x) \geqq \varepsilon-2 \lambda=\varepsilon^{\prime}$. Let $k$ be an integer such that $\left|3 f_{N}^{n}(x) / \varepsilon^{\prime}-k\right|<1$. Then $\varepsilon^{\prime}(k-1) / 3$ $<f_{N}^{n}(x)<\varepsilon^{\prime}(k+1) / 3$ and $f_{N}^{n+m}(x) \geqq \varepsilon^{\prime}+\varepsilon^{\prime}(k-1) / 3=\varepsilon^{\prime}(k+2) / 3$.

Similarly, if $f^{n}(x)-f^{n+m}(x) \leqq \varepsilon$ for some nonnegative integers $n$ and $m$ and for some $x$ in $X$ such that $\max \left[\left|g_{N}^{v}(x)\right|: 0 \leqq \nu \leqq n+m\right]<\lambda$ then there exists a nonnegative integer $k$ such that $f_{N}^{n+m}(x) \leqq k \varepsilon^{\prime} / 3$ and $f_{N}^{n}(x) \geqq(k+1) \varepsilon^{\prime} / 3$.

Let $M$ be any nonnegative integer such that $(M+1) \varepsilon^{\prime} / 3 \geqq N$ and let $\lambda$ be any real number with $0<\lambda<\varepsilon^{\prime} / 6$. Then from the reasoning above we conclude

$$
\begin{aligned}
\mu\left(\left\{x: \max \left[\left|f^{k}(x)-f^{j}(x)\right|\right.\right.\right. & : n \leqq j<k \leqq m+n]>\varepsilon\}) \\
& \leqq \sum_{k=0}^{M} \mu\left(\left\{x: U_{n, m}^{*}\left(x \mid f_{N}^{k}, \frac{k \varepsilon^{\prime}}{3}+\lambda, \frac{(k+1) \varepsilon^{\prime}}{3}-\lambda\right)>0\right\}\right)+\frac{\varepsilon}{2}
\end{aligned}
$$

for every pair of nonnegative integers $n$ and $m$.

By Lemma 4 , for each integer $k$ with $0 \leqq k \leqq M$, there is a nonnegative $n_{k}$ such that

$$
\mu\left(\left\{x: U_{n, m}^{*}\left(x \mid f_{N}^{k}, k \varepsilon^{\prime} / 3+\lambda,(k+1) \varepsilon^{\prime} / 3-\lambda\right)>0\right\}\right)<\varepsilon / 2(M+1)
$$

for each pair of nonnegative integers $n$ and $m$ with $n \geqq n_{k}$.

Define $\bar{n}=\max \left[n_{k}: 0 \leqq k \leqq M\right]$. Then if $n$ and $m$ are any nonnegative integers with $n \geqq \bar{n}$,

$$
\mu\left(\left\{x: \max \left[\left|f^{k}(x)-f^{j}(x)\right|: n \leqq j \leqq k \leqq n+m\right]>\varepsilon\right\}\right)<\varepsilon .
$$

Let $\left\{\varepsilon_{n}\right\}_{n=1}^{\infty}$ be any sequence of positive real numbers for which $\lim _{n \rightarrow \infty} \varepsilon_{n}$ exists and is equal to 0 . For each pair of nonnegative integers $n$ and $m$ define the set

$$
A_{n, m}=\left\{x: \max \left[\left|f^{k}(x)-f^{j}(x)\right|: n \leqq j \leqq k \leqq n+m\right]>\varepsilon_{n}\right\} .
$$

We have shown above that $\lim _{n \rightarrow \infty} \mu\left(A_{n, m}\right)=0$ uniformly for nonnegative integers $m$. 
Since

$$
0 \leqq \mu\left(A_{n, m+k}\right)-\mu\left(A_{n, m}\right) \leqq \mu\left(A_{n+m, k}\right)
$$

for all nonnegative integers $n, m$, and $k$ we have

$$
\lim _{m \rightarrow \infty}\left|\mu\left(A_{n, m+k}\right)-\mu\left(A_{n, m}\right)\right|=0
$$

uniformly for nonnegative integers $k$, for each nonnegative integer $n$. Therefore the sequence $\left\{\mu\left(A_{n, m}\right)\right\}_{m=0}^{\infty}$ is a Cauchy sequence for each nonnegative integer $n$. Define the set $A_{n}=\bigcup\left\{A_{n, m}: 0 \leqq m<\infty\right\}$ for each nonnegative integer $n$. Since the sequence $\left\{\mu\left(A_{n, m}\right)\right\}_{m=0}^{\infty}$ is a Cauchy sequence, it follows that $A_{n}$ is integrable and $\lim _{m \rightarrow \infty} \mu\left(A_{n, m}\right)=\mu\left(A_{n}\right)$ for each nonnegative integer $n$.

Define the set $A=\bigcap\left\{A_{n}: 0 \leqq n<\infty\right\}$. Since $\lim _{n \rightarrow \infty} \mu\left(A_{n, m}\right)=0$ uniformly for nonnegative integers $m$ it follows that $A$ is integrable and

$$
\mu(A)=\lim _{n \rightarrow \infty} \mu\left(A_{n}\right)=0 .
$$

Therefore the sequence $\left\{f^{n}\right\}$ is Cauchy almost everywhere.

Now assume that (1.1) holds.

If $f^{*}$ is the almost everywhere limit of the sequence of averages $\left\{f^{n}\right\}$ of a bounded integrable function $f$, Lebesgue's Dominated Convergence Theorem [1, p. 200] implies that $f^{*} \cdot I_{A}$ is integrable whenever $I_{A}$ is the characteristic function of an integrable set and, moreover,

$$
\lim _{n \rightarrow \infty} \int_{A} f^{n} d \mu=\int_{A} f^{*} d \mu .
$$

A straightforward argument based on Lemma 1 and the uniform integrability of the sequence $\left\{f^{n}\right\}$ together with the convergence theorems in [1, pp. 197-200] generalizes this result to not necessarily bounded functions.

It follows directly from this that if $E$ is invariant under $T$ (i.e. if $I_{E} \circ T=I_{E}$ a.e.) if $A$ is any integrable set, and if $f \geqq 0$ is any integrable function,

$$
\int_{E} f d \mu \geqq \int_{A \cap E} f^{*} d \mu
$$

Let $A$ and $B$ be integrable sets and let $\varepsilon>0$. Let $\delta>0$ be such that

$$
2 \delta+\sqrt{ }(2 \delta) \max \{\mu(A)+\mu(B), 1\}<\varepsilon / 2 .
$$

Inductively define sequences $\left\{A_{k}: 1 \leqq k<\infty\right\},\left\{B_{k}: 1 \leqq k<\infty\right\},\left\{A_{k}^{\prime}: 0 \leqq k<\infty\right\}$, and $\left\{B_{k}^{\prime}: 0 \leqq k<\infty\right\}$ of integrable sets and a sequence $\{t(k): 1 \leqq k<\infty\}$ of integers so that $T^{t(k)} A_{k}=B_{k}(k \geqq 1)$ and there are disjoint partitions

$$
\begin{aligned}
A & =A_{1} \cup \cdots \cup A_{k} \cup A_{k}^{\prime} & & (k \geqq 1), \\
B_{k}^{\prime} & =B_{1} \cup \cdots \cup B_{k} \cup B & & (k \geqq 1),
\end{aligned}
$$


and the following inequality is satisfied:

$$
\mu\left(B_{k}^{\prime} \cap T^{t(k+1)} A_{k}^{\prime}\right)<\int_{B_{k}^{\prime}} I_{k}^{*} d \mu+\delta \quad(k \geqq 0)
$$

where $I_{k}^{*}$ is the almost everywhere limit of the averages $\left\{I_{k}^{n}\right\}$ of the characteristic function $I_{k}$ of $A_{k}^{\prime}$, by proceeding as follows.

Let $A_{0}^{\prime}=A$ and $B_{0}^{\prime}=B$. Having found the required sets and integers up to index $k \geqq 1$, let $N \geqq 0$ be any integer so that

$$
\left|\int_{B_{k-1}^{\prime}} I_{k-1}^{N} d \mu-\int_{B_{k-1}^{i}} I_{k-1}^{*} d \mu\right|<\delta .
$$

Then

$$
\frac{1}{N+1} \sum_{j=0}^{N}\left\{\int_{B_{k-1}^{\prime}} T^{j} I_{k-1} d \mu-\int_{B_{k-1}^{\prime}} I_{k-1}^{*} d \mu-\delta\right\}<0
$$

so that, for some integer $j$, with $0 \leqq j \leqq N$,

$$
\int_{B_{k-1}^{\prime}} T^{j} I_{k-1} d \mu=\mu\left(B_{k-1}^{\prime} \cap T^{-j} A_{k-1}^{\prime}\right)<\int_{B_{k-1}^{\prime}} I_{k-1}^{*} d \mu+\delta .
$$

Let $t(k)=j$ and define the integrable sets

$$
\begin{array}{ll}
B_{k}=T^{-j} A_{k-1}^{\prime}-B_{k-1}^{\prime}, & A_{k}=T^{j} B_{k}, \\
B_{k}^{\prime}=B_{k-1}^{\prime} \cup B_{k}, & A_{k}^{\prime}=A_{k-1}^{\prime}-A_{k} .
\end{array}
$$

Now let $n$ be any integer for which $\mu(A)<n \cdot \delta$. Then

$$
\begin{aligned}
n \cdot \delta>\mu(A) & \geqq \sum_{k=1}^{n} \mu\left(A_{k}\right) \geqq \sum_{k=1}^{n} \mu\left(T^{-t(k)} A_{k-1}^{\prime}-B_{k-1}^{\prime}\right) \\
& \geqq \sum_{k=0}^{n-1}\left[\mu\left(A_{k}^{\prime}\right)-\int_{B_{k}} I_{k}^{*} d \mu-\delta\right]
\end{aligned}
$$

or

$$
\sum_{k=0}^{n-1}\left[\mu\left(A_{k}^{\prime}\right)-\int_{B_{k_{k}^{\prime}}} I_{k}^{*} d \mu-2 \delta\right]<0
$$

so that for some integer $K, 0 \leqq K \leqq n-1$,

$$
\mu\left(A_{K}^{\prime}\right)<\int_{B_{K}^{\prime}} I_{K}^{*} d \mu+2 \delta .
$$

Define $E=\left\{x: I_{K}^{*}(x)>\sqrt{ }(2 \delta)\right\}$.

To show $E$ is integrable let $n \geqq 0$ be integer and $E^{\prime}$ an integrable set so that $\left|I_{K}^{*}(x)-I_{k}^{n}(x)\right|<\frac{1}{2} \sqrt{ }(2 \delta)$ if $x \in-E^{\prime}$ and $\mu\left(E^{\prime}\right)<1$.

Then $E \subset E^{\prime \prime}=\left\{x: I_{k}^{n}(x)>\frac{1}{2} \sqrt{ }(2 \delta)\right\} \cup E^{\prime}$ and $E^{\prime \prime}$ is integrable. Since a measurable 
subset of an integrable set is integrable, it follows that $E$ is integrable. It is an easy consequence of the properties of Césaro averages that $E$ is invariant. Consequently

$$
\mu\left(A_{K}^{\prime} \cap E\right)=\int_{E} I_{K} d \mu \geqq \int_{E} I_{K}^{*} d \mu .
$$

Define the sets

$$
\begin{array}{ll}
A_{0}=A_{K}^{\prime}-E, & B_{0}=E-B_{K}^{\prime}, \\
A_{*}=A_{K}^{\prime} \cap E, & B_{*}=-E-B_{K}^{\prime} .
\end{array}
$$

Since $E$ is invariant, we have $T^{k}\left(A_{K}^{\prime} \cap E\right) \cap B_{*}=\varnothing$ for all integers $k$. In addition (5) and (6) imply

$$
\begin{aligned}
\mu\left(A_{0}\right) & =\mu\left(A_{K}^{\prime}-E\right)=\mu\left(A_{K}^{\prime}\right)-\mu\left(A_{K}^{\prime} \cap E\right) \\
& <\int_{B_{K}^{\prime}} I_{K}^{*} d \mu+2 \delta-\int_{E} I_{K}^{*} d \mu \\
& <2 \delta+\int_{B_{K}^{\prime}-E} I_{K}^{*} d \mu \leqq 2 \delta+\sqrt{ }(2 \delta) \mu\left(B_{K}^{\prime}\right) \\
& <2 \delta+\sqrt{ }(2 \delta)(\mu(A)+\mu(B))<\varepsilon / 2
\end{aligned}
$$

while (5) and (6) also imply

$$
\int_{B_{K}^{\prime}} I_{K}^{*} d \mu+2 \delta>\int_{E} I_{K}^{*} d \mu
$$

so that

$$
2 \delta>\int_{E-B_{K}^{\prime}} I_{R}^{*} d \mu \geqq \sqrt{ }(2 \delta) \mu\left(E-B_{K}^{\prime}\right)
$$

and

$$
\mu\left(B_{0}\right)=\mu\left(E-B_{K}^{\prime}\right)<\sqrt{ }(2 \delta)<\varepsilon / 2 .
$$

It follows that the partition $A=A_{0} \cup A_{1} \cup \cdots \cup A_{K} \cup A_{*},-B=B_{0} \cup B_{1} \cup \ldots$ $\cup B_{K} \cup B_{*}$, together with the integers $t(1), \ldots, t(K)$ satisfy the condition (1.2).

COROLlaRY 1. For a measure preserving transformation $T$ on a set which has $a$ subset of positive measure, constructively equivalent are

(2.1) for each integrable function $f$, the sequence of averages $\left\{f^{n}\right\}$ converges a.e. to a constant, and

(2.2) for each pair of integrable sets $A$ and $B$ and for every $\varepsilon>0$ there are disjoint integrable partitions $A=A_{0} \cup A_{1} \cup \cdots \cup A_{n},-B=B_{0} \cup B_{1} \cup \cdots \cup B_{n}$ and integers $t(1), \ldots, t(n)$ such that $T^{t(k)} A_{k}=B_{k}(1 \leqq k \leqq n)$ such that $T^{k} A_{*} \cap B_{*}=\varnothing$ for all integers $k$, and such that at least one of $A_{0}$ and $B_{0}$ is integrable with measure less than $\varepsilon$.

Proof. The proof that (2.1) implies (2.2) is identical to the proof that (1.1) implies (1.2) with the additional observation that if the limit function $I_{K}^{*}$ is constant a.e., then the integrable set $E$ or its complement may be taken to be a null set and thus either $A_{*}$ or $B_{*}$ is null.

To show (2.2) implies (2.1), first consider a nonnegative bounded integrable function $f$. Without loss of generality assume $0 \leqq f \leqq 1$ a.e. Since (2.2) implies (1.2), 
the proposition implies the sequence of averages $\left\{f^{n}\right\}$ converges a.e. to a measurable function $f^{*}$. Since $0 \leqq f^{n} \leqq 1$ a.e. it follows that $0 \leqq f^{*} \leqq 1$ a.e. Let $\varepsilon>0$. We now construct sequences of real numbers $\left\{\alpha_{j}\right\}$ and $\left\{\beta_{j}\right\}$ to satisfy the inequalities $\alpha_{j} \leqq \alpha_{j+1}$ $<\beta_{j+1} \leqq \beta_{j}$ and $\left(\beta_{j+1}-\alpha_{j+1}\right) \leqq \frac{2}{3}\left(\beta_{j}-\alpha_{j}\right)$ and also, except on a set of measure less than $\varepsilon\left(1-2^{-j}\right)$, the inequality $\alpha_{j} \leqq f^{*} \leqq \beta_{j}$, these to hold for all positive integers $j$. Set $\alpha_{1}=0$ and $\beta_{1}=1$. Let $n$ be a positive integer. If $\alpha_{1} \leqq \cdots \leqq \alpha_{n}<\beta_{n} \leqq \cdots \leqq \beta_{1}$ have been defined, let $a$ and $b$ be any real numbers such that $\frac{2}{3} \alpha_{n}+\frac{1}{3} \beta_{n} \leqq a<b \leqq \frac{1}{2} \alpha_{n}+\frac{2}{3} \beta_{n}$ and such that the sets $A=\left\{x: f^{*}(x) \leqq a\right\}$ and $B=\left\{x: f^{*}(x) \geqq b\right\}$ are measurable. The sets $B$ and $-A$ are integrable. Thus by (2.2) and by the fact $\mu\left(A \cap T^{j} B\right)=0$ for all integers $j$, we have either $\mu(B)<\varepsilon / 2^{n+1}$ or $A$ is integrable and $\mu(A)<\varepsilon / 2^{n+1}$.

In the first case take $\alpha_{n+1}=\alpha_{n}$ and $\beta_{n+1}=b$. In the second take $\alpha_{n+1}=a$ and $\beta_{n+1}=\beta_{n}$. Then in either event $\alpha_{n} \leqq \alpha_{n+1}<\beta_{n+j} \leqq \beta_{n}$ and $\left(\beta_{n+1}-\alpha_{n+1}\right) \leqq \frac{2}{3}\left(\beta_{n}-\alpha_{n}\right)$ and $\alpha_{n+1} \leqq f^{*} \leqq \beta_{n+1}$ except on a set of measure less than $\varepsilon\left(1-2^{-n}+2^{-n-1}\right)$ $=\varepsilon\left(1-2^{-n-1}\right)$.

Having completed the inductive definition, it is trivial that $\left\{\alpha_{j}\right\}$ and $\left\{\beta_{j}\right\}$ are Cauchy converging to a common limit $L_{\varepsilon}$ and $f^{*}(x)=L_{\varepsilon}$ for all $x$ except in an integrable set $E_{\varepsilon}$ of measure less than $\varepsilon$. Suppose $A$ is a set of positive measure in $X$; then $L_{\varepsilon}=L_{\varepsilon^{\prime}}$ whenever $0<\varepsilon, \varepsilon^{\prime}<\frac{1}{2} \mu(A)$. Thus $f^{*}$ is constant a.e.

By linearity, the result follows for all bounded integrable functions $f$ and if $f$ is not necessarily bounded, Lemma 1 implies the result in a straightforward fashion.

COROLlary 2. Let $T$ be a measure preserving transformation such that for all integrable functions $f$ the averages $\left\{f^{n}\right\}$ converge a.e. to a measurable function $f^{*}$. Then constructively equivalent are

(3.1) for each integrable function $f$, the a.e. limit $f^{*}$ of the averages $\left\{f^{n}\right\}$ is integrable; and

(3.2) for every increasing sequence $\left\{X_{q}\right\}$ of integrable subsets whose characteristic functions converge almost everywhere to 1 , the following holds:

for every integrable set $A$ and $\varepsilon>0$ there is a disjoint partition into integrable sets $A=A_{1} \cup A_{2}$ such that

(i) there is an integer $q$ such that, for all integers $k, \mu\left(T^{k} A_{1}-X_{q}\right)<\varepsilon$ and

(ii) for all integers $p$ there is an integer $N$ such that $\mu\left(A_{2}-\bigcup\left\{T^{k}\left(X_{p}\right):|k| \leqq N\right\}\right)<\varepsilon$.

Proof. To show (3.2) implies (3.1) it is sufficient (by linearity) to consider the case of a nonnegative integrable function $f$.

Let $\left\{X_{q}^{\prime}: 0 \leqq q<\infty\right\}$ be an increasing sequence of integrable subsets of $X$ whose characteristic functions converge to 1 a.e.

We shall show that the sequence of real numbers $\left\{\int_{X_{p}} f^{*} d \mu\right\}$ is Cauchy and it will follow from the monotone convergence theorem that $f^{*}$ is integrable.

Let $\varepsilon>0$. By the monotone convergence theorem

$$
\lim _{q \rightarrow \infty} \int_{X_{q}^{\prime}} f d \mu=\int f d \mu .
$$


Let $q_{0}$ be so large that $\int_{-A} f d \mu<\varepsilon$ for $A \equiv X_{q_{0}}^{\prime}$ and let $\delta>0$ be so small that if $E$ is an integrable set for which $\mu(E)<\delta$ then $\int_{E} f d \mu<\varepsilon$.

Define a sequence $\left\{X_{q}\right\}$ of integrable subsets of $X$ inductively as follows. Let $X_{0}=0$. Having defined $X_{q}$ for $0 \leqq k \leqq n$, let $A=A_{0} \cup A_{c} \cup A_{*},-X=Y_{0} \cup Y_{c} \cup Y_{*}$ be disjoint measurable partitions of $A$ and $-X_{n}$ so that $\mu\left(A_{0}\right)+\mu\left(Y_{0}\right)<\delta / 4$, the sets $T^{k} A_{*} \cap Y_{*}=\varnothing$ and $T: A_{c} \rightarrow Y_{c}$, where the latter symbolism means that there are disjoint integrable partitions $A_{c}=A_{1} \cup \ldots \cup A_{n}, \quad Y_{c}=Y_{1} \cup \cdots \cup Y_{n}$ and integers $t(1), \ldots, t(n)$ such that $T^{t(k)} A_{k}=Y_{k}(k=1, \ldots, n)$.

Define $X_{n+1}=X_{q_{0}+n+1}^{\prime} \cup Y_{0} \cup Y_{c} \cup X_{n}$.

The sequence $\left\{X_{p}\right\}$ thus defined is increasing and converges a.e. to 1. In light of (3.2) there is an integrable partition $A=A_{1} \cup A_{2}$ such that

(i) there is an integer $q \geqq 0$ for which $\mu\left(T^{k} A_{1}-X_{q}\right)<\delta / 4$ for all integers $k$, and

(ii) for every integer $p \geqq 0$ there is an integer $N \geqq 0$ such that

$$
\mu\left(A_{2}-\bigcup\left\{T^{k}\left(-X_{p}\right):|k| \leqq N\right\}\right)<\delta / 4 .
$$

Let $p \geqq q$ be an integer. We now show that

$$
\int_{X_{p}} f^{*} d \mu-\int_{X_{q}} f^{*} d \mu<8 \varepsilon
$$

Let $f=f_{1}+f_{2}+f_{3}$ where $f_{i}=f \cdot I_{A_{i}}$ for $i=1$ or 2 and $f_{3}=f \cdot I_{-A}$. Then $f^{*}=f_{1}^{*}+f_{2}^{*}$ $+f_{3}^{*}$ a.e. First

$$
\int_{X_{p}-X_{q}} f_{3}^{*} d \mu \leqq \int_{X_{p}} f_{3}^{*} d \mu \leqq \int f_{3} d \mu=\int_{A} f d \mu<\varepsilon
$$

by choice of $A \equiv X_{q_{0}}^{\prime}$. Now let $N$ be so large that

Then from

$$
\int_{X_{p}-X_{q}} f_{1}^{*} d \mu<\int_{X_{p}-X_{q}} f_{1}^{N} d \mu+\varepsilon
$$

$$
\int_{X_{p}-x_{q}} f_{1}^{N} d \mu \leqq \frac{1}{N+1} \sum_{k=0}^{N} \int_{-X_{q} \cap T-k_{A_{1}}} T^{k} f d \mu
$$

and $\mu\left(-X_{q} \cap T^{-k} A_{1}\right)<\delta$ for all $k$, and from the choice of $\delta$ it follows that

$$
\int_{X_{p}-X_{q}} f_{1}^{*} d \mu<\left(\frac{1}{N+1} \sum_{k=0}^{N} \varepsilon\right)+\varepsilon=2 \varepsilon
$$

Finally to show $\int f_{2}^{*} d \mu<5 \varepsilon$ we construct a sequence $\left\{q_{i}\right\}$ of integers as follows. Let $q_{0}=q$ and $q_{1}=p$. Having defined $q_{0}, \ldots, q_{n}$ so that

$$
\int_{X_{q_{k}}-X_{q_{k-1}}} f_{2}^{*} d \mu>\int_{X_{p}-X_{q}} f_{2}^{*} d \mu-4 \varepsilon
$$

we proceed to construct measurable partitions $-X_{q_{n}}=X_{0}^{\prime} \cup X_{c}^{\prime} \cup X_{*}^{\prime}, X_{p}-X_{q}$ $=X_{0}^{\prime \prime} \cup X_{c}^{\prime \prime} \cup X_{*}^{\prime \prime}$ where $\mu\left(X_{0}^{\prime}\right)+\mu\left(X_{0}^{\prime \prime}\right)<\delta / 4$, the sets $T^{k} X_{*} \cap X_{*}^{\prime \prime}=\varnothing$ for all integers $k$, and $T: X_{c}^{\prime} \rightarrow X_{c}^{\prime \prime}$. 
Let $q_{n+1}$ be an integer such that $\mu\left(X_{c}^{\prime}-X_{q_{n+1}}\right)<\delta / 4$. Then

$$
\int_{X_{q_{n}+1}-X_{q_{n}}} f_{2}^{*} d \mu>\int_{X_{c}^{\prime}} f_{2}^{*} d \mu-\varepsilon>\int_{X_{c}^{\prime \prime}} f_{2}^{*} d \mu-\varepsilon \geqq \int_{X_{p}-X_{q}} f_{2}^{*} d \mu-2 \varepsilon-\int_{X_{*}^{*}} f_{2}^{*} d \mu .
$$

To complete the induction it is therefore enough to show $\int_{X_{*}^{\prime \prime}} f_{2}^{*} d \mu<2 \varepsilon$ and for this it is enough to show $\mu\left(X_{*}^{\prime \prime} \cap T^{k} A_{2}\right)<\delta$ for all integers $k$, since then an argument identical to that used to estimate $\int_{X_{p}-X_{q}} f_{1}^{*} d \mu$ will give the desired inequality.

By construction of the sequence $\left\{X_{q}\right\}$ there are disjoint measurable partitions $-X_{q_{n+1}}=Y_{0} \cup Y_{c} \cup Y_{*}$ and $A=A_{0} \cup A_{c} \cup A_{*}$ where $Y_{*} \cap T^{j} A_{*}=\varnothing$ for all integers $j$, and $\mu\left(Y_{0}\right)+\mu\left(A_{0}\right)<\delta / 4$, and $T: A_{c} \rightarrow Y_{c}$, and additionally $Y_{*} \supset X_{\varnothing}$ where $\tilde{q} \equiv q_{n+1}+1$. Let $N$ be an integer such that $\mu\left(A_{2}-\bigcup\left\{T^{j}\left(-X_{q}\right):|j| \leqq N\right\}\right)$ $<\delta / 4$.

Then $\delta / 4>\mu\left(A_{2} \cap A_{*}-\bigcup\left\{T^{j}\left(-X_{q}\right):|j| \leqq N\right\}\right)=\mu\left(A_{2} \cap A_{*}\right)$. Consequently

$$
\mu\left(X_{*}^{\prime \prime} \cap T^{k} A_{2}\right)<\delta / 2+\mu\left(X_{*}^{n} \cap T^{k}\left(A_{2} \cap A_{c}\right)\right) .
$$

For some integrable subset $Y_{c}^{\prime} \subset Y_{c} \subset-X_{q_{n+1}}$ we have $T:\left(T^{-k} X_{*}^{\prime \prime} \cap A_{2} \cap A_{c}\right) \rightarrow Y_{c}^{\prime}$. Since $X_{*}^{\prime \prime} \cap T^{j} X_{*}^{\prime}=\varnothing$ for all integers $j$, we must have $Y_{c}^{\prime} \subset X_{0}^{\prime} \cup\left(X_{c}^{\prime}-X_{q_{n+1}}\right)$. Thus

$$
\mu\left(X_{k}^{\prime \prime} \cap T^{k}\left(A_{2} \cap A_{c}\right)\right)<\mu\left(X_{0}^{\prime} \cup\left(X_{c}^{\prime}-X_{q_{n+1}}\right)\right)<\delta / 2 .
$$

Putting all together, $\mu\left(X_{k}^{\prime \prime} \cap T^{k} A_{2}\right)<\delta$ as was to be shown.

This completes the inductive definition of the sequence $\left\{q_{k}\right\}$ and for $k \geqq 1$

$$
\int_{X_{q_{k}}-X_{q_{k-1}}} f_{2}^{*} d \mu>\int_{X_{p}-X_{q}} f_{2}^{*} d \mu-4 \varepsilon
$$

Summing this inequality for $k=1, \ldots, n$ yields

$$
\int_{X_{q_{n}}-X_{q}} f_{2}^{*} d \mu>n\left(\int_{X_{p}-X_{q}} f_{2}^{*} d \mu-4 \varepsilon\right)
$$

and so, for all integers $n \geqq 1$,

$$
\int_{X_{p}-X_{q}} f_{2}^{*} d \mu-4 \varepsilon<\frac{1}{n} \int_{X_{p_{n}}} f_{2}^{*} d \mu \leqq \frac{1}{n} \int f d \mu .
$$

Taking $n$ so large that $(1 / n) \int f d \mu<\varepsilon$, it follows that $\int_{X_{p}-X_{q}} f_{2}^{*} d \mu<5 \varepsilon$. Consequently, for the given $\varepsilon>0$ and the modified sequence $\left\{X_{p}\right\}$ there is an integer $q$ such that, for $p \geqq q, \int_{X_{p}-X_{q}} f^{*} d \mu<8 \varepsilon$. Let $r$ be an integer such that $\mu\left(X_{q}-X_{r}^{\prime}\right)<\delta$. Thus for suitably large integers $n$

$$
\int_{X_{q}-X_{r}^{\prime}} f^{*} d \mu<\int_{X_{q}-X_{r}^{\prime}} f^{n} d \mu+\varepsilon<\frac{1}{n+1} \sum_{0}^{n}(\varepsilon)+\varepsilon=2 \varepsilon
$$

and consequently since $X_{p+q_{0}}^{\prime} \subset X_{p}$ we have, for $p \geqq q$,

$$
\int_{X_{p+q_{0}}^{\prime}-X_{r}^{\prime}} f^{*} d \mu \leqq \int_{X_{p}-X_{q}} f^{*} d \mu+\int_{X_{q}-X_{r}^{\prime}} f^{*} d \mu<10 \varepsilon
$$


It follows that the sequence $\left\{\int_{X_{p}^{\prime}} f^{*} d \mu\right\}$ is Cauchy and therefore that $f^{*}$ is integrable.

To prove (3.1) implies (3.2), let $\left\{X_{q}\right\}$ be an increasing sequence of subsets of $X$, whose characteristic functions converge to 1 a.e.

Let $A$ be an integrable set and let $\varepsilon>0$. Let $q_{0}$ be an integer such that $q \geqq q_{0}$ implies, for $f=I_{A}, \int_{X_{q}-X_{q_{0}}} f^{*} d \mu<\varepsilon / 5$.

Let $A_{1}=\left\{x: f^{*}(X) \geqq \varepsilon / 5 \mu\left(X_{q_{0}}\right)\right\}$ and let $q$ be an integer such that $\mu\left(A_{1} \cap X_{q}\right)$ $>\mu\left(A_{1}\right)-\varepsilon / 5$. Then $\mu\left(T^{k} A_{1}-X_{q}\right)<\varepsilon$ for all integers $k$ showing that (i) of (3.2) is satisfied. Let $p \geqq q_{0}$. Choose an integer $n$ such that

$$
\int_{X_{p}-X_{q_{0}}} f^{n} d \mu<\int_{X_{p}-X_{q_{0}}} f^{*} d \mu+\varepsilon / 5 \text { and } \int_{X_{q_{0}}-A_{1}} f^{n} d \mu<\int_{X_{q_{0}}-A_{1}} f^{*} d \mu+\varepsilon / 5 \text {. }
$$

Then $\int_{X_{p}-X_{q_{0}}} f^{n} d \mu<2 \varepsilon / 5$. We can write

$$
\mu(A)=\int f d \mu=\int f^{n} d \mu=\int_{-X_{p}} f^{n} d \mu+\int_{X_{p}-X_{q_{0}}} f^{n} d \mu+\int_{X_{q_{0}}} f^{n} d \mu .
$$

Setting $A_{2} \equiv A-A_{1}$ and $f_{i}=I_{A_{i} \cap A}$ for $i=1$ or 2 it follows that

$$
\int_{-x_{p}} f_{2}^{n} d \mu>\mu\left(A_{2}\right)+I_{2}-I_{3}-I_{4}-2 \varepsilon / 5
$$

where

$$
I_{2}=\mu\left(A_{1} \cap A\right)-\int_{X_{q_{0}}} f_{1}^{n} d \mu \geqq \mu\left(A_{1} \cap A\right)-\int f_{1}^{n} d \mu=0
$$

and

$$
I_{3}=\int_{X_{q_{0}}} f_{2}^{n} d \mu=\int_{X_{q_{0}}-A_{1}} f_{2}^{n} d \mu<\int_{X_{q_{0}}-A_{1}} f^{*} d \mu+\varepsilon / 5<2 \varepsilon / 5
$$

and finally

$$
I_{4}=\int_{-X_{p}} f_{1}^{n} d \mu=\frac{1}{n+1} \sum_{0}^{n} \mu\left(T^{-k}\left(A \cap A_{1}\right)-X_{p}\right) \leqq \frac{1}{n+1} \sum_{0}^{n}(\varepsilon / 5)=\varepsilon / 5 .
$$

Thus

$$
\frac{1}{n+1} \sum_{0}^{n} \mu\left(T^{-k} A_{2}-X_{p}\right)=\int_{-x_{p}} f_{2}^{n} d \mu>\mu\left(A_{2}\right)-\varepsilon
$$

so that for some integer $k, 0 \leqq k \leqq n, \mu\left(T^{-k} A_{2}-X_{p}\right)>\mu\left(A_{2}\right)-\varepsilon$ and so part (ii) of (3.2) is satisfied.

COROLlaRY 3. If $T$ is a measure preserving transformation on a finite measure space then equivalent are

(4.1) for every integrable function $f$, the sequence of averages $\left\{f^{n}\right\}$ converges a.e. to $1 /(\mu(X)) \int f d \mu$, 
(4.2) there is a nonnegative monotone increasing function $r$ defined for nonnegative real numbers such that

(i) for all $\varepsilon>0$ there is $a \delta>0$ so that $x<\varepsilon$ if $r(x)<\delta$, and

(ii) for every pair of integrable sets $A$ and $B$ and every $\varepsilon>0$ there is an integer $k$ for which

$$
\mu\left(A \cap T^{k} B\right)>r(\mu(A) \cdot \mu(B))-\varepsilon .
$$

Proof. Without loss of generality take $\mu(X)=1$.

Assume (4.1). Let $A$ and $B$ be integrable sets and let $\varepsilon>0$. Since $\left\{I_{A}^{n}\right\}$ converges a.e. to $\mu(A)$, there is an integer $N \geqq 0$ such that $\int_{B}\left(\mu(A)-I_{A}^{N}\right) d \mu<\varepsilon$. Thus

$$
\frac{1}{N+1} \sum_{k=0}^{N}\left(\mu\left(B \cap T^{-k} A\right)-\mu(B) \mu(A)-\varepsilon\right)<0
$$

which directly implies (4.2) with $r(x)=x$.

Assume (4.2). We first show that (2.2) holds.

Let $A$ and $B$ be integrable sets and let $\varepsilon>0$. Let $\delta>0$ such that $x<\varepsilon^{2}$ if $r(x)<2 \delta$. By induction construct a sequence $\left\{n_{k}\right\}_{k=1}^{\infty}$ of integers and sequences of disjoint integrable subsets $\left\{A_{k}\right\}_{k=1}^{\infty}$ of $A$ and $\left\{B_{k}\right\}_{k=1}^{\infty}$ of $B$ as follows.

Let $n_{1}=0$ and $A_{1}=B_{1}=A \cap B$. Having constructed integers $\left\{n_{v}: 1 \leqq \nu<k\right\}$ and sets $\left\{A_{v}: 1 \leqq \nu<k\right\}$ and $\left\{B_{v}: 1 \leqq \nu<k\right\}$ for some integer $k>1$, define

$$
\bar{A}_{k}=A-\bigcup\left\{A_{v}: 1 \leqq \nu<k\right\}, \quad \bar{B}_{k}=B-\bigcup\left\{B_{v}: 1 \leqq \nu<k\right\} .
$$

By hypothesis, there is an integer $t$ such that $\mu\left(\bar{A}_{k} \cap T^{t} \bar{B}_{k}\right)>r\left(\mu\left(\bar{A}_{k}\right) \mu\left(\bar{B}_{k}\right)\right)-\delta$. Let $n_{k}$ be this integer and define $A_{k}=\bar{A}_{k} \cap T^{t} \bar{B}_{k}, B_{k}=T^{-t} A_{k}$.

Having completed the induction, let $K$ be a positive integer so large that $\mu(A) / K<\delta$. Then, as a consequence of the induction construction,

$$
\begin{aligned}
\mu(A) & \geqq \sum_{k=2}^{K+1} \mu\left(A_{k}\right)>\sum_{k=2}^{K+1}\left[r\left(\mu\left(\bar{A}_{k}\right) \mu\left(\bar{B}_{k}\right)\right)-\delta\right] \\
& >K\left[r\left(\mu\left(\bar{A}_{K+1}\right) \mu\left(\bar{B}_{K+1}\right)\right)-\delta\right] .
\end{aligned}
$$

That is $\delta>\mu(A) / K>r\left(\mu\left(\bar{A}_{K+1}\right) \mu\left(\bar{B}_{K+1}\right)\right)-\delta$ and therefore $r\left(\mu\left(\bar{A}_{K+1}\right) \mu\left(\bar{B}_{K+1}\right)\right)<2 \delta$. By the choice of $\delta$, this implies

$$
\left[\min \left\{\mu\left(\bar{A}_{K+1}\right) \mu\left(\bar{B}_{K+1}\right)\right\}\right]^{2} \leqq \mu\left(\bar{A}_{K+1}\right) \mu\left(\bar{B}_{K+1}\right)<\varepsilon^{2}
$$

and therefore that at least one of $\mu\left(\bar{A}_{K+1}\right)$ and $\mu\left(\bar{B}_{K+1}\right)$ is less than $\varepsilon$.

Define $A_{0}=\bar{A}_{k+1}, B_{0}=\bar{B}_{K+1}$. It follows that the partitions $A=A_{0} \cup \cup_{k=1}^{K} A_{k}$, $B=B_{0} \cup \bigcup_{k=1}^{K} B_{k}$ together with the integers $n_{1}, \ldots, n_{K}$ satisfy the requirements of (2.2). Therefore (4.2) implies (2.2).

Let $f$ be an integrable function. By Corollary 2, the sequence of averages $\left\{f^{n}\right\}$ converges a.e. to a constant $\alpha$. Consequently, since the space $X$ is an integrable set and $\mu(X)=1$

$$
\int f d \mu=\lim _{n \rightarrow \infty} \int f^{n} d \mu=\alpha
$$




\section{REFERENCES}

1. E. Bishop, Foundations of constructive analysis, McGraw-Hill, New York, 1967, pp. 153242. MR 36 \#4930.

2. - Mathematics as a numerical language (to appear).

3. N. Dunford and J. T. Schwartz, Linear operators. I: General theory, Pure and Appl. Math., vol. 7, Interscience, New York, 1958, pp. 675-676. MR 22 \#8302.

Department of Mathematics, University of Miami, Coral Gables, Florida 33124 